\title{
Supplement:
}

\section{Business Ecosystems and Platforms: Towards a Shared Understanding}

\author{
Günter Müller-Stewens \& Joachim Stonig
}

Cite as: Müller-Stewens, G. \& Stonig, J. 2019. Business Ecosystems and Platforms: Towards a Shared Understanding. Die Unternehmung, 73(4): 381-386.

The term ecosystem is currently attracting enormous interest among managers, consultants and researchers. Unfortunately, in some areas it has also become a fashionable "buzzword" used in an almost inflationary way. As a result, our understanding of what exactly an ecosystem isand what it is not - in a business context has expanded considerably as well.

We have therefore taken the authors' conference on this special issue as an opportunity to seek a common understanding of the key terms used in the context of ecosystems.

Business ecosystems are an organizational form that enables coordination between independent but complementary players around a shared value proposition, thus creating added value.

Based on this definition, the following characteristics of a business ecosystem emerge:

- “organizational form": A business ecosystem is an organizational form for economic transactions between different players and is therefore an own entrepreneurial unit. ${ }^{1}$ However, this does not necessarily mean that an ecosystem must act in a profit-oriented manner-primarily socially oriented transactions can also be organized by an ecosystem.

- “around a shared value proposition": The basis of the ecosystem is a shared value proposition that addresses a specific customer need. ${ }^{2}$ This value proposition represents the mission of the ecosystem, as it defines its purpose. Ecosystems are therefore configured based on a customer need to be served.

A special feature of ecosystems is that they can serve even very complex customer needs through the coordinated interaction of the various participants. The value proposition offers a solution to a customer problem, which is based on an integrated and comprehensive offering, but at the same time has a high degree of user-friendliness (convenience).

This distinguishes ecosystems, for example, from "virtual organizations", which are configured more from the point of view of firms, such as a group of industrial companies that have expanded their spread of offerings through a network. ${ }^{3}$

- “enables coordination": Performance in an ecosystem is based on the possibility and ability of breaking down a complex value proposition into independent activity modules in such a way that these function independently, but at the same time interact in a coordinated manner. The activities on which the value proposition is based are thus configured ${ }^{4}$ in a new way — sometimes spanning industry boundaries.

The coordination of activities in an ecosystem is managed by an orchestrator that assumes a central role for the players cooperating in the ecosystem (therefore sometimes also referred

\footnotetext{
1 Also see Jacobides/Cennamo/Gawer (2018).

${ }^{2}$ Also see Kapoor (2018).

${ }^{3}$ See Müller-Stewens (editor, 1997).

${ }^{4}$ Also see Adner (2017).
} 
to as a lead organization). The orchestrator, for example, ensures the development of the common value proposition and defines the rules of interaction (via technological interfaces, revenue mechanisms, etc.). The orchestrator thus exerts a certain degree of control over the ecosystem. The role of the orchestrator is not always assumed by a single organization. Often there is a technical operator of the ecosystem and an owner-as in the case of smartphones, where companies like Google/Alphabet control the software platform of the ecosystem, but the technical infrastructure is produced by device manufacturers like Samsung.

- "complementary": The activities underlying the components of the integrated solution are performed by complementors. The term complementor (in contrast to terms like ecosystem partner) emphasizes that the components in the ecosystem are complementary, i.e., without them the ecosystem does not function or functions worse. The activities of the complementors are coordinated by the orchestrator, such that their offerings fit together in a modular way. In some ecosystems, complementors of the same activity compete with each other. However, value creation in an ecosystem is not always based on having as many complementors as possible; success often depends on the availability of the complementors that are essential from the customer's point of view. The interaction between customers and complementors is direct, the orchestrator only facilitates these interactions. However, this does not rule out the possibility that the orchestrator itself may offer certain complements, thus taking a double role.

The users or customers of an ecosystem are usually not just passive consumers. In order to fulfill an ecosystem's complex value proposition, users can be highly involved and interact intensively with the ecosystem, e.g., by delegating responsibility or providing data. They often take on the role of complementors themselves (for instance, by creating content). Just like complementors, users therefore also contribute to the ecosystem value.

- “independent players": The players involved join the ecosystem on their own initiative and can leave it again of their own accord, taking possible contracts into account. Of course, there may be implicit constraints, but there is still freedom of choice. This is one of the differences to diversified corporations to which the subsidiaries are legally bound e.g., to a holding company.

In contrast to more vertically organized value chains ("pipelines"), ecosystems are based on horizontal, network-like cooperation in which the participants meet on an equal footing. So there is no tendency towards subordination. Since not all details of the cooperation can be specified using contracts, the establishment of an underlying basis of trust between the participants is important. This demands a considerable degree of collaboration competence from the participating organizations. If this is not forthcoming, it will not be possible to attract suitable complementors for one's own ecosystem and keep them in the system. This means that the ability to interact in partnerships is critical for the ecosystem participants, especially for orchestrators.

- "creating added value": The economic goal of a business ecosystem is that the value of the ecosystem is greater than the sum of its parts (the activities of orchestrators and complementors), i.e., an ecosystem surplus is achieved. This surplus is generated by various value drivers, in particular network effects and learning effects. Network effects occur when a larger or broader range of complementary partners has a positive influence on customers' willingness to join and pay. Learning effects mean that the ecosystem can offer a better value proposition through a deeper and longer interaction between the different participants, which in turn positively influences willingness to pay. 
This added value can manifest itself as financial profit for the players. However, it is not certain that the orchestrator and complementors will appropriate this added value; it can also be the customers (e.g., in the case of Uber, which is currently not making a profit despite an attractive customer offering). The definition of the ecosystem boundaries is also crucial for measuring added value. For example, a bike sharing system, as such, may not create financial added value, but it can indirectly create added value for a city's mobility ecosystem through spill-over effects.

In the context of business ecosystems, there is often talk of "platforms" or "platform-based business models". It should be noted here that not every platform-based business model is also an ecosystem. Conversely, not every business model of an ecosystem is technologically implemented via a platform, even though this is frequently the case (as platforms considerably accelerate the scalability of the business model and thus also lead to the realization of network effects).

\section{Platforms are technological infrastructures connecting players and objects.}

- "technological infrastructure": In its simplest form, a platform is nothing more than a technological foundation that brings together different activities (of players and objects) in a coordinated way. The field of application is extremely broad and can range from a motherboard in a computer, through a dating website, up to a platform construction kit in the automotive industry

- “connecting": A platform must create the conditions for platform participants to interact efficiently together. For this purpose, the platform operator defines interfaces that ensure that the various activities of all players mesh together as seamlessly as possible. However, networking is not only based on an efficient technological infrastructure (hardware and software), but also on social norms and rules of interaction (e.g., entry and exit prerequisites, conflict escalation mechanisms, revenue mechanics and calculation modes, etc.). ${ }^{5}$

Digital platforms are particularly suitable for the implementation of ecosystems because many elements of value creation processes can now be networked via digital interfaces. This makes it easier for business ecosystems to work together beyond organizational and industry boundaries and significantly reduces transaction costs between those involved. IT platforms have thus become a key enabler of many of today's business ecosystems.

The activities connected via the platform and suitable interfaces become modules that can be easily exchanged and changed without affecting the entire platform. This results in a platform architecture, usually with a stable platform core and flexible connected modules. ${ }^{6}$

With regard to the networking and openness of the platform in relation to the modules, a distinction is made between one-sided and two-sided platforms. Two (or more) sided platforms bring together the modules of different sites, e.g., providers and users. A classic example here is Ebay or a dating platform. The presence on one side has an effect on the other side, which is not completely internalized by the participants. ${ }^{7}$ In contrast, there are

\footnotetext{
${ }^{5}$ Also see Nambisan/Sawhney (2011) or Wareham/Fox/Giner (2014).

${ }^{6}$ Also see Baldwin/Woodard (2009) and Gawer (2014).

${ }^{7}$ Also see Rochet/Tirole (2006). A participant from one side does not consider its own contribution to the value of the platform when making a purchase decision. For example: A man who joins a dating platform takes only the ratio of the supply of women to the cost of the platform as a basis for his purchase decision. However, he does not take into account the fact that his purchase decision increases the value of the platform for the women's side and so he should therefore pay less.
} 
one-sided platforms where platform participants do not interact with another side-like a supply chain platform in the automotive industry.

- "players": In its simplest form of connecting players, a platform is a virtual electronic marketplace. The players and users of a platform are then the sellers and their buyers/customers. Here it is irrelevant whether networking takes place at the consumer-toconsumer (C2C), peer-to-peer (P2P), business-to-consumer (B2C) or business-to-business (B2B) level.

The platform assumes a mediating function between the players. Information that enables more efficient networking is therefore critical to the success of the platform. Bringing together supply and demand gives rise to transactions that generate data. Platforms that have a larger number of participants and a higher volume of usage data are usually more successful. A platform can also selectively pass on data to participants, e.g., to developers who receive access to user data, advance information on interface changes or shares of sales from the use of the app.

- “objects": Platforms can network not only players, but also objects. In industrial platforms, for instance, the Internet of Things (IoT) forms the technological basis for networking. Physical products, such as machines or semi-finished products, become information carriers that generate data from built-in sensors in order to control processes via the platform. One step further, however, they may also be able to initiate follow-up activities in production processes independently or automatically (smart devices). For system manufacturers (such as lift systems), communication with the systems after delivery can also be ensured in this way, e.g., in order to identify maintenance requirements at an early stage (predictive maintenance).

\section{Platform-based business models are mechanisms building on platforms for value creation and distribution. ${ }^{8}$}

- “mechanisms building on platforms": Digital platforms enable new business models with very high scalability and scope. Thanks to extensive standardization and automation of the transaction process between the participants, only very low transaction costs are incurred. The computing capacity required is not a real bottleneck, so the marginal costs of growth are often close to zero. The essential question is to what extent investors are prepared to finance the investment costs of the first few years associated with high negative cash flows.

- "value creation": If the focus of the platform is primarily on establishing transactions, then value arises here (1) through the transactions of the players involved, which are brought together, and (2) through the provision of the information necessary for transactions to occur.

The platform can also generate additional value, e.g., (3) through services to support the execution of transactions (such as payment processing or mutual evaluation of players), and (4) by providing and safeguarding the legal and regulatory framework conditions.

A key aspect of value generation (for multi-sided platforms) is the network effects to be expected with the use of a platform. This means that with a growing number of participants, the benefit for the players on both sides of the platform increases (dis)proportionally. This results from feedback effects: The more transactions there are, the more attractive it is to participate - whether because of greater choice or higher availability. This means that the greater the number of networked players on the platform and the more data generated, the

\footnotetext{
${ }^{8}$ For the definition of business models, see Zott/Amit/Massa (2011).
} 
greater the value of the platform. This applies both to network effects among users of the same side (same-side effect) and to network effects between different sides, because the platform is all the more attractive for providers the more buyers there are and vice versa (cross-side effect). The network effects, however, only comes about above a critical mass of users and providers.

Network effects can be enhanced by the skillful use of data. In the case of transactions on the platform, the players involved generate data which they (consciously or unconsciously) make available to the operator of the platform. Additional value is created if knowledge about user behavior is gained from this transaction data and passed on to the providers, as appropriate. Using algorithms, often based on artificial intelligence methods, tailored offers and recommendations can be created for individual customers.

For example, a fintech company can use a platform to submit personalized investment proposals to the user via its own app after linking to the user's bank account. As bank data also provides information on other preferences and behaviors, personalized offerings beyond banking (e.g., mobility services) can be made using additional apps based on the available user data. Often users can also download such additional functions via a "store". This can also take place in a form in which the user does not directly recognize that the offer comes from third parties (white label solution).

If it is possible to make the platform attractive for particularly efficient providers, this could open up great potential for innovation. Companies such as Microsoft, Google and Apple hold large developer conferences every year. At the Apple Worldwide Developers Conference (WWDC), for example, an outlook will be given on the next version of the operating systems for Apple devices. This should motivate developers to develop innovative new functions for the devices. In certain regions, such as Silicon Valley, there are real innovation clusters of such developers.

- "value distribution": The distribution of the value creation of a platform-based business model takes place between the platform and the participating sides on the one hand, and between competing platforms, on the other. The former depends on the distribution of power between the different sides, which influences the collection of fees and the distribution of data. The possibility of players being active on several platforms at the same time (multi-homing) or circumventing them plays a role in the distribution of power. The latter is based on the results of platform competition. The presence of network effects often leads to an oligopolization of global platform markets (winner-takes-all effect).

If one follows the above explanations, it is easy to see that ecosystems and platforms have many things in common, but are still not the same. Ecosystems are an inter-company organizational form that addresses a value proposition; platforms are a technical infrastructure that can be used, but does not have to be used, for the operational implementation of ecosystems. An ecosystem can, for example, consist of several (nested) platforms.

In both cases, firms need to think carefully about their role in a changing competitive environment: Do they want to set up and operate such an ecosystem or platform themselves, or do they only want to participate as a complementor?

\section{References}

Adner, R. (2017): Ecosystem as Structure: An Actionable Construct for Strategy, in: Journal of Management, Vol. 43, No. 1, p. 39-58. 
Baldwin, C. Y./Woodard, C. J. (2009): The Architecture of Platforms: A Unified View, in Gawer, A. (ed.) Platforms, Markets and Innovation. Cheltenham, U.K. and Northampton, Mass.: Elgar.

Gawer, A. (2014): Bridging Differing Perspectives on Technological Platforms: Toward an Integrative Framework, in: Research Policy, Vol. 43, No. 7, p. 1239-1249.

Jacobides, M. G./Cennamo, C./Gawer, A. (2018): Towards a Theory of Ecosystems, in: Strategic Management Journal, Vol. 39, No. 8, p. 2255-2276.

Kapoor, R. (2018): Ecosystems: Broadening the Locus of Value Creation, in: Journal of Organization Design, Vol. 7, No. 1, p. 12.

Müller-Stewens, G. (ed.). (1997): Die Virtualisierung von Organisationen, Schäffer-Poeschel Verlag/Verlag NZZ: Stuttgart/Zürich.

Nambisan, S./Sawhney, M. (2011): Orchestration Processes in Network-Centric Innovation: Evidence From the Field, in: Academy of Management Perspectives, Vol. 25, No. 3, p. 40-57.

Rochet, J.-C./Tirole, J. (2006): Two-Sided Markets: a Progress Report, in: RAND Journal of Economics, Vol. 37, No. 3, p. 645-667.

Wareham, J./Fox, P. B./Giner, J. L. C. (2014): Technology Ecosystem Governance, in: Organization Science, Vol. 25, No. 4, p. 1195-1215.

Zott, C./Amit, R./Massa, L. (2011): The Business Model: Recent Developments and Future Research, in: Journal of Management, Vol. 37, No. 4, p. 1019-1042. 